\title{
Anestesia peridural com lidocaína isolada ou associada à clonidina: efeito cardiorrespiratório e analgésico em cães
}

\author{
Epidural anesthesia with lidocaine alone or combinated with clonidine: cardiopulmonary and \\ analgesic effects in dogs
}

\author{
Renata Navarro Cassu ${ }^{*}$ Alessandra Melchert ${ }^{\mathrm{II}}$ Gláucia Moreno Machado ${ }^{\mathrm{III}}$ \\ Carlos Collares Meirelles ${ }^{\mathrm{I}}$
}

\section{RESUMO}

Analgesia satisfatória tem sido relatada com a administração peridural de agonistas adrenérgicos em associação aos anestésicos locais. Objetivou-se, com este trabalho, avaliar o efeito analgésico e cardiorrespiratório da lidocaína isolada ou associada à clonidina via peridural lombossacra em cães. Seis cães foram submetidos a dois tratamentos, com intervalo mínimo de 15 dias entre cada avaliação. No tratamento L, foi empregada lidocaína $2 \%$ com vasoconstrictor $\left(5 \mathrm{mg} \mathrm{kg}^{-1}\right)$ e, no tratamento $C$, a clonidina $\left(10 \mu \mathrm{g} \mathrm{kg}^{-1}\right)$ foi associada à lidocaína, de modo a perfazer um

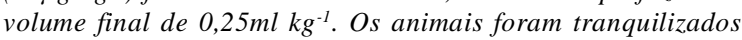
com acepromazina $\left(0,05 \mathrm{mg} \mathrm{kg}^{-1} \mathrm{IV}\right)$ e mantidos sob anestesia com isofluorano em máscara facial durante a punção do espaço peridural. Foram mensuradas: frequência cardíaca $(F C)$, parâmetros eletrocardiográficos $(E C G)$, frequência respiratória (f), pressão arterial sistólica (PAS), gases sanguíneos, temperatura retal $(T)$, duração e extensão do bloqueio anestésico. A estatística foi realizada com análise de variância, teste de Tukey e teste t pareado $(P<0,05)$. A FC foi inferior aos 15, 30 e 45 minutos após a anestesia peridural, no tratamento $C$, em relação ao $L$. Arritmias não foram observadas em nenhum dos tratamentos. A PAS, a f, a T e os gases sanguíneos não diferiam entre os grupos. A duração do bloqueio anestésico não variou entre os tratamentos, sendo de $71 \pm 13$ e $75 \pm 26$ minutos, nos tratamentos $L$ e $C$, respectivamente. Com relação à extensão do bloqueio, a altura máxima atingida foi a quarta vértebra lombar e a décima vértebra torácica, nos tratamentos L e C, respectivamente. Conclui-se que a associação da clonidina à lidocaína promoveu bloqueio anestésico mais cranial, quando comparado ao uso isolado do anestésico local, porém induziu bradicardia, além de não prolongar a duração do bloqueio anestésico.

Palavras- chave: anestésico local, $\alpha_{2}$ agonista, extradural, cão.

\begin{abstract}
Satisfactory analgesia has been related with epidural $\alpha_{2}$ adrenoceptor agonists in combination with local anesthetics. The aim of this study was to compare the analgesic and cardiopulmonary effects of lidocaine or lidocaine-clonidine epidural injections in healthy dogs. Dogs were randomly assigned to two groups of six animals each. The L group received lidocaine $\left(5 \mathrm{mg} \mathrm{kg}^{-1} \mathrm{~L}\right)$ and the $C$ group lidocaine plus clonidine $\left(10 \mu \mathrm{kg}^{-1} \mathrm{C}\right)$. Preanaesthetic medication was carried out with acepromazine $\left(0.05 \mathrm{mg} \mathrm{kg}^{-1} \mathrm{IV}\right)$. Anaesthesia was induced and maintained with isoflurane by facial mask for epidural injection. Heart rate $(H H)$, electrocardiography $(E C G)$, respiratory rate $(R R)$, systolic arterial blood pressure (SAP), rectal temperature $(R T)$, blood gases, duration of anesthesia and sensitive block level were investigated. Statistical analysis was performed with ANOVA, Tukey test and paired t test. There was a decrease of $H R$ at 15, 30 and 45 minutes after epidural injection in $C$ group when compared with $L$ group. Cardiac dysrhythmias did not observe in both treatments. The variables, SAP, RR, RT and blood gases did not differ between treatments. Duration of anesthesia was $71 \pm 13$ and $75 \pm 26$ minutes to $L$ and $C$ treatment, respectively, without difference between groups. The maximal extension of sensitive block was the fourth lumbar vertebra and the tenth thoracic vertebra in $L$ and $C$ treatment, respectively. In conclusion, the addition of clonidine to lidocaine in epidural anesthesia allowed more cranial sensory block in relation to the isolated use of local anesthetic, however, it produced bradycardia and did not prolong the duration of anesthesia.
\end{abstract}

Key words: local anesthetic, $\alpha_{2}$-agonist, extradural, dog.

'Departamento de Cirurgia e Anestesiologia Veterinária (DCAV), Faculdade de Ciências Agrárias (FCA), Universidade do Oeste Paulista (Unoeste), Rodovia Raposo Tavares, Km 572, Campus II, Bairro Limoeiro, 19067-175, Presidente Prudente, SP, Brasil. E-mail: renavarro@uol.com.br.*Autor para correspondência.

"Departamento de Clínica Médica de Pequenos Animais, FCA, Unoeste, Presidente Prudente, SP, Brasil.

${ }^{\text {IIIC } C u r s o ~ d e ~ M e d i c i n a ~ V e t e r i n a ́ r i a, ~ F C A, ~ U n o e s t e, ~ P r e s i d e n t e ~ P r u d e n t e, ~ S P, ~ B r a s i l . ~}$ 


\section{INTRODUÇÃO}

A associação de agonistas alfa adrenérgicos com anestésicos locais por via peridurał tem sido proposta para uso tanto em homens, como em animais, em função da possibilidade do incremento do efeito analgésico e do aumento da duração e da extensão do bloqueio anestésico (EISENACH et al., 1989; IVANI et al.,2000; BRONDANI et al., 2004).

A clonidina é um composto imidazólico

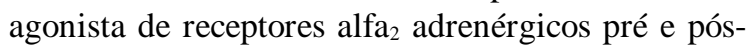
sinápticos com propriedades ansiolíticas e hipnóticas. Quando administrada por via peridural, produz analgesia, sem bloqueio motor, por meio de um mecanismo de ação nãoopioide, com ausência de efeitos adversos como náusea, prurido ou depressão respiratória (BOPP, 2004; SISTO, 2010).

Em ovelhas, a administração peridural de clonidina produziu efeitos analgésicos intensos com mínima depressão cardiorrespiratória (EISENACH et al., 1987), corroborando os resultados obtidos por GHIGNONE et al. (1987), em estudo similar realizado em cães, no qual foi demonstrado intenso efeito analgésico, com mínimas alterações hemodinâmicas e respiratórias.

Assim, objetivou-se, com este trabalho, avaliar os efeitos cardiorrespiratório e analgésico mediados pela lidocaína isolada ou associada à clonidina em cães. Paralelamente, foi investigada a possibilidade do aumento da extensão cranial e da duração do bloqueio anestésico em função da adição da clonidina à lidocaína.

\section{MATERIAL E MÉTODOS}

Foram avaliados seis cães, provenientes do canil da instituição de origem, sem raça definida, com peso médio de $7,1 \pm 1 \mathrm{~kg}$, clinicamente saudáveis, avaliados por meio de exame físico e laboratorial (hemograma). Os mesmos animais foram utilizados nos dois tratamentos, com intervalo mínimo de 15 dias entre cada experimento. No tratamento L, foi empregada lidocaína $2 \%$ com vasoconstritor ${ }^{\mathrm{a}}\left(5 \mathrm{mg} \mathrm{kg}^{-1}\right)$ e, no tratamento $\mathrm{C}$, a clonidina ${ }^{\mathrm{b}}\left(10 \mu \mathrm{g} \mathrm{kg}^{-1}\right)$ foi associada à lidocaína $2 \%$ com vasoconstritor, de modo a perfazer um volume final de $0,25 \mathrm{ml} \mathrm{kg}^{-1}$.

Todos os cães foram submetidos a jejum alimentar e hídrico de 12 e 4 horas, respectivamente, antes da realização do experimento. A medicação préanestésica (MPA) foi realizada com acepromazina ${ }^{c}$ $\left(0,05 \mathrm{mg} \mathrm{kg}^{-1} \mathrm{IV}\right)$. Vinte minutos após, os animais foram induzidos e mantidos em anestesia inalatória com isofluorano ${ }^{\mathrm{d}}$ em máscara facial, atingindo-se plano anestésico superficial, durante o período da punção do espaço peridural (L7-S1). Os animais foram posicionados em decúbito esternal, estendendo-se os membros pélvicos, cranialmente para a punção do espaço peridural, cuja localização foi confirmada pela aspiração de uma gota do anestésico, depositada no canhão da $\operatorname{agulha}^{\mathrm{e}}$ (CRUZ et al., 1997). A injeção peridural foi realizada em período de 60 segundos, momento em que a anestesia inalatória foi interrompida.

Foram avaliados: frequência cardíaca (FC) e eletrocardiograma $(\mathrm{ECG})^{\mathrm{f}}$ nas derivações bipolares (I, II e II) e unipolares aumentadas (avR, avL e avF), na velocidade de $50 \mathrm{~mm} \mathrm{seg}^{-1}$ e calibração da milivoltagem em $1 \mathrm{~cm}=1 \mathrm{mV}$; ; pressão arterial sistólica $(\mathrm{PAS})^{\mathrm{g}}$, pelo método não invasivo, com adaptação do manguito no membro torácico direito, acima da articulação do carpo, respeitando-se uma relação de 0,4 entre a largura do manguito e a circunferência do membro; frequência respiratória $(f)$, mediante a observação dos movimentos torácicos em um minuto; temperatura retal (T), com o emprego de termômetro clínico digital ${ }^{\mathrm{h}}$, posicionado na ampola retal durante 2 minutos; pressão parcial de dióxido de carbono no sangue arterial $\left(\mathrm{PaCO}_{2}\right)$, pressão parcial de oxigênio no sangue arterial $\left(\mathrm{PaO}_{2}\right)$, saturação de oxigênio na hemoglobina $\left(\mathrm{Sat}_{\mathrm{O} 2}\right)$, $\mathrm{pHe}$ bicarbonato $\left(\mathrm{HCO}_{3}^{-1}\right)$, mediante punção da artéria femoral, com agulha hipodérmica $13 \times 4,5$, em seringa previamente heparinizada, com avaliação em equipamento específicoi.

AFC, o ECG e a PAS foram avaliados antes da MPA (basal), 15 minutos após a MPA e a cada 15 minutos após a injeção peridural. A T foi avaliada antes da MPA (basal), 15 minutos após a MPA e a cada 30 minutos após a injeção peridural. A colheita de sangue arterial para hemogasometria foi realizada aos 15 minutos após a MPA (basal) e a cada 30 minutos após a anestesia peridural. Todas as variáveis foram aferidas com os animais completamente recuperados da anestesia inalatória, até a obtenção do retorno do reflexo interdigital.

A duração do bloqueio anestésico foi avaliada com estímulos nociceptivos somáticos, aplicados a cada 15 minutos, na prega interdigital dos membros pélvicos, imediatamente após a mensuração das variáveis fisiológicas. O estímulo foi aplicado com a pinça Kelly, cujas ranhuras foram protegidas por borracha, sendo realizado o pinçamento da prega interdigital até a primeira cremalheira da pinça ou até resposta positiva do animal, detectada pelo movimento de retirada do membro. O mesmo estímulo foi empregado para avaliar a analgesia somática, com aplicação na região dorsal, iniciando-se na área lombossacra, em direção cranial às vértebras lombares e torácicas, até 
ser identificada a resposta positiva do animal, detectada por movimentos de cabeça, com direcionamento da face em direção à área estimulada, tentativas de morder a pinça, possíveis gemidos ou latidos durante a aplicação do estímulo somático. Essa avaliação permitiu a identificação da extensão do bloqueio anestésico, uma vez que os estímulos nociceptivos foram realizados em áreas anatômicas específicas, sendo registrado o ponto na coluna vertebral, em que o animal apresentava sensibilidade (GASPARINI et al., 2007; CASSU et al., 2010).

A estatística foi realizada por meio de análise de variância (ANOVA) para amostras pareadas (repeated measure ANOVA), com contrastes pelo método de Tukey para avaliação de diferenças ao longo do tempo dentro de cada grupo e teste " $\mathrm{t}$ " pareado para comparação das diferenças entre os grupos, em cada momento, considerando-se a diferença significativa quando $\mathrm{P}<0,05$ (ZAR, 1996).

\section{RESULTADOS}

Houve redução significativa na FC após a peridural, no tratamento $\mathrm{C}$, em relação aos valores basais, enquanto que, no tratamento L, esse parâmetro não apresentou alteração significativa ao longo do tempo. $\mathrm{Na}$ comparação entre os grupos, valores inferiores foram observados aos 15, 30 e 45 minutos após a peridural, no tratamento $C$ (Tabela 1). Além da bradicardia, nenhuma outra alteração de ritmo foi detectada em nenhum dos grupos.

Houve redução significativa na $f$ após a anestesia peridural, em relação aos valores basais a partir dos 15 e dos 30 minutos, nos tratamentos L e C,

Tabela 1 - Valores médios e desvio padrão da frequência cardíaca (FC), frequência respiratória $(f)$, pressão parcial de dióxido de carbono no sangue arterial $\left(\mathrm{PaCO}_{2}\right)$, pressão parcial de oxigênio no sangue arterial $\left(\mathrm{PaO}_{2}\right), \mathrm{pH}$, saturação de oxigênio na hemoglobina $\left(\mathrm{Sa}_{\mathrm{tO} 2}\right)$ e no bicarbonato $\left(\mathrm{HCO}_{3}^{-}\right)$, pressão arterial sistólica (PAS) e da temperatura retal (T), em cães sob anestesia peridural com lidocaína isolada $(\mathrm{L}, \mathrm{n}=6)$ ou associada à clonidina $(\mathrm{C}, \mathrm{n}=6)$.

\begin{tabular}{|c|c|c|c|c|c|c|c|}
\hline Variável/Grupos & Basal & MPA & $15 \mathrm{~min}$ & $30 \mathrm{~min}$ & $45 \mathrm{~min}$ & $60 \mathrm{~min}$ & $75 \mathrm{~min}$ \\
\hline \multicolumn{8}{|c|}{ - } \\
\hline $\mathrm{L}$ & $100 \pm 11$ & $94 \pm 37$ & $104 \pm 39$ & $89 \pm 30$ & $91 \pm 28$ & $86 \pm 24$ & --------- \\
\hline $\mathrm{C}$ & $97 \pm 12$ & $94 \pm 21$ & $56 \pm 15^{* \#}$ & $56 \pm 13^{* \#}$ & $61 \pm 13^{* \#}$ & $59 \pm 14^{*}$ & $53 \pm 12^{*}$ \\
\hline & & & $-\mathrm{f}(\operatorname{mov} n$ & 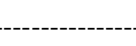 & & & -------- \\
\hline $\mathrm{L}$ & $33 \pm 9$ & $22 \pm 4$ & $15 \pm 3 *$ & $18 \pm 5^{*}$ & $18 \pm 5^{*}$ & $15 \pm 4 *$ & ---------- \\
\hline $\mathrm{C}$ & $26 \pm 5$ & $24 \pm 6$ & $18 \pm 7$ & $13 \pm 5^{*}$ & $15 \pm 6^{*}$ & $17 \pm 6^{*}$ & $18 \pm 4 *$ \\
\hline & & & $-\mathrm{PaCO}_{2}(\mathrm{n}$ & (----- & & & ----- \\
\hline $\mathrm{L}$ & ---------- & $35 \pm 4$ & ----------- & $35 \pm 4$ & ----------- & $37 \pm 5$ & -------- \\
\hline $\mathrm{C}$ & --------- & $33 \pm 5$ & ---------- & $36 \pm 4$ & --------- & $36 \pm 9$ & --------- \\
\hline & & & $-\mathrm{PaO}_{2}(\mathrm{~m}$ & ----------- & (200 & & - \\
\hline $\mathrm{L}$ & ---------- & $99 \pm 11$ & ---------- & $100 \pm 10$ & ---------- & $102 \pm 5$ & ---------- \\
\hline $\mathrm{C}$ & --------- & $109 \pm 38$ & ---------- & $103 \pm 11$ & --------- & $101 \pm 11$ & ---------- \\
\hline & & & $-\mathrm{pH}$ (unic & 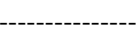 & & - & -------- \\
\hline $\mathrm{L}$ & --------- & $7,39 \pm 0,03$ & --------- & $7,40 \pm 0,01$ & --------- & $7,35 \pm 0,02$ & --------- \\
\hline $\mathrm{C}$ & --------- & $7,44 \pm 0,03$ & --------- & $7,41 \pm 0,02$ & --------- & $7,36 \pm 0,06$ & --------- \\
\hline-- & & & $-\mathrm{Sa}_{\mathrm{tO} 2}$ & . & 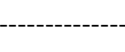 & 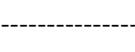 & ---------- \\
\hline $\mathrm{L}$ & ---------- & $98 \pm 0,02$ & ---------- & $98 \pm 0,03$ & ---------- & $98 \pm 0,03$ & ---------- \\
\hline $\mathrm{C}$ & ---------- & $97 \pm 0,02$ & --------- & $97 \pm 0,03$ & ---------- & $98 \pm 0,08$ & ---------- \\
\hline & & & $-\mathrm{HCO}_{3}^{-}(\mathrm{mr}$ & - & & ---------- & -------- \\
\hline $\mathrm{L}$ & ---------- & $20 \pm 1$ & --------- & $20 \pm 0,7$ & --------- & $20 \pm 0,6$ & --------- \\
\hline $\mathrm{C}$ & --------- & $21 \pm 1$ & ---------- & $21 \pm 1,1$ & --------- & $22 \pm 1,3$ & ---------- \\
\hline $\mathrm{L}$ & $144 \pm 12$ & $120 \pm 24$ & $\begin{array}{l}--P A S(m \\
144 \pm 57\end{array}$ & $126 \pm 28$ & $134 \pm 21$ & $123 \pm 21$ & - \\
\hline $\mathrm{C}$ & $143 \pm 41$ & $116 \pm 36$ & $110 \pm 39^{*}$ & $118 \pm 59$ & $101 \pm 33^{*}$ & $109 \pm 33^{*}$ & $107 \pm 18^{*}$ \\
\hline $\mathrm{L}$ & $38,6 \pm 0,3$ & $38,4 \pm 0,2$ & -------- & $37,6 \pm 0,2 *$ & --------- & $37,8 \pm 0,2 *$ & ------- \\
\hline $\mathrm{C}$ & $39,1 \pm 1,1$ & $38,8 \pm 0,8$ & --------- & $37,4 \pm 0,3^{*}$ & --------- & $36,8 \pm 0,4^{*}$ & --------- \\
\hline
\end{tabular}

*Diferença significativa em relação ao valor basal; ${ }^{*}$ Diferença significativa entre os tratamentos $(\mathrm{P}<0,05)$. 
respectivamente, perdurando até o término da avaliação. Não houve diferença entre os grupos para essa variável (Tabela 1).

Com relação aos gases sanguíneos $\left(\mathrm{PaCO}_{2}\right.$, $\mathrm{PaO}_{2} \mathrm{eSat}_{\mathrm{O} 2}$ ), ao $\mathrm{pHe}$ ao $\mathrm{HCO}_{3}$, não foram observadas diferenças entre os grupos e nem ao longo do tempo (Tabela 1).

A PAS não variou ao longo do tempo, nos animais do tratamento L. No tratamento C, houve redução significativa na PAS, em relação aos valores basais aos 15, 45, 60 e 75 minutos após a peridural. Não foram observadas diferenças na PAS entre os grupos (Tabela 1).

Em ambos os grupos, foi observada redução na temperatura retal após a anestesia peridural em relação aos valores basais (Tabela 1).

A duração do bloqueio anestésico não diferiu entre os grupos, com tempo médio de $71 \pm 13$ e $75 \pm 26$ minutos, nos tratamentos Le C, respectivamente. Com relação à dispersão do bloqueio, a altura máxima atingida foi até a sétima vértebra lombar, em dois animais, e até a quarta vértebra lombar, no tratamento $\mathrm{L}$, enquanto que, no tratamento $\mathrm{C}$, três animais apresentaram ausência de sensibilidade até a primeira vértebra lombar, um animal até a segunda vértebra lombar e os outros dois animais até a décima vértebra torácica.

\section{DISCUSSÃO}

A clonidina, quando administrada via peridural, atua em receptores alfa ${ }_{1}$ e, preferencialmente, em receptores alfa ${ }_{2}$ adrenérgicos situados no corno dorsal da medula espinhal (EISENACH et al., 1987), mediando analgesia dependente da dose (De KOCK et al., 1997). No cão, há uma grande divergência em relação à dose da clonidina para uso peridural, de modo que a dose empregada no atual estudo baseou-se em resultados prévios (GHIGNONE et al., 1987; BOPP, 2004; ROSO et al., 2005), visando a uma dose que fosse capaz de promover analgesia, com mínimos efeitos adversos.

O estímulo dos receptores alfa adrenérgicos determinado pela clonidina, além de mediar analgesia, também é responsável pela redução do tônus simpático medular e pelo incremento do tônus parassimpático, podendo resultar em depressão hemodinâmica (GHIGNONE et al., 1987). No atual estudo, foi observada bradicardia ( $\mathrm{FC}<60$ batimentos minutos $^{-1}$ ) nos animais tratados com clonidina, corroborando os resultados relatados por ROSO et al. (2005). A bradicardia é decorrente da ativação dos adrenoceptores alfa pré-sinápticos, resultando na redução da exocitose da noradrenalina e do efeito simpatolítico sobre o sistema nervoso central, induzido pela clonidina (De KOCK et al., 1997). Paralelamente, a bradicardia induzida pela clonidina também está relacionada à ativação dos receptores imidazolínicos, situados no núcleo reticular (TIBIRIÇA et al., 1991). Contudo, apesar da bradicardia, a clonidina não determinou alteração de ritmo cardíaco, o que concorda com resultados prévios relatados no cão (SISTO, 2010). Existem controvérsias em relação ao efeito da clonidina na fisiologia cardíaca. Alguns autores relatam que a clonidina não altera o ritmo cardíaco (TOUSSAINT et al., 1984), enquanto outros sugerem que esse fármaco reduz a velocidade de condução do estímulo elétrico cardíaco (CLEMENTY et al., 1986). Dessa forma, a clonidina deve ser evitada em animais portadores de cardiopatias, pois, em pacientes predispostos, esse fármaco pode desencadear arritmias (EISENACH et al., 1989). Por outro lado, os animais tratados com lidocaína isolada, apesar de também não terem apresentado arritmias, mantiveram a FC dentro da normalidade para a espécie, resultados que concordam com estudos prévios realizados no cão (GASPARINI et al., 2007; CASSU et al., 2010).

A pressão arterial sistólica declinou após a administração peridural de clonidina em relação aos valores basais, porém hipotensão não foi observada, corroborando resultados prévios relatados no homem (DE KOCK et al., 1997), no cão (BOPP, 2004; ROSO et al., 2005) e em ovinos (EISENACH \& GRICE, 1988). O mecanismo de ação da clonidina sobre a pressão arterial é complexo. A clonidina tem ação espinhal e supraespinhal, o que resulta na redução da pressão arterial, mas paralelamente atua nos receptores alfa adrenérgicos periféricos localizados nos vasos 2 sanguíneos, culminando com vasoconstricção (EISENACH et al., 1987). Dessa forma, tem sido demonstrado que o uso de doses altas de clonidina induz à menor redução da pressão arterial, quando comparado ao uso de doses menores, em função do incremento do estímulo dos receptores alfa adrenérgicos (EISENACH \& GRICE, 1988). Paralelamente, os animais tratados com lidocaína isolada também demonstraram estabilidade na PAS, resultado que concorda com estudos prévios (CASSU et al., 2008; CASSU et al., 2010).

A frequência respiratória reduziu em ambos os grupos após a anestesia peridural, porém os valores observados mantiveram-se dentro dos limites fisiológicos para a espécie, não tendo relevância clínica. Ademais, foi observada estabilidade nos gases sanguíneos durante todo o período de avaliação, com valores de $\mathrm{PaCO}_{2}, \mathrm{PaO}_{2}$, $\mathrm{Sat}_{\mathrm{O} 2}$ e $\mathrm{pH}$ dentro dos valores fisiológicos para a espécie, comprovando a ausência 
de depressão respiratória em ambos os grupos. Resultados semelhantes foram descritos em cães, após administração peridural de clonidina (BOPP, 2004; SISTO, 2010) ou lidocaína isolada (NOLTE et al., 1983; CASSU et al., 2008; CASSU et al., 2010).

A discreta redução da temperatura retal observada em ambos os tratamentos, ao longo do tempo, pode ser atribuída à ação vasodilatadora e depressora dos mecanismos termorreguladores do hipotálamo decorrente da acepromazina (BROCK, 1994). Adicionalmente, a anestesia peridural também prejudica o controle da termorregulação central e periférica, favorecendo a ocorrência da hipotermia (MATSUKAWA et al., 1995). Apesar de não ter sido observada alteração importante desse parâmetro, a redução da temperatura foi um pouco mais pronunciada nos animais do tratamento $\mathrm{C}$, provavelmente devido à depressão central, bem como à redução da atividade muscular, determinadas pela clonidina (SINCLAIR, 2003).

O incremento da duração do bloqueio peridural pela adição da clonidina aos anestésicos locais tem sido relatado no homem (IVANI et al., 2000; DE NEGRI et al., 2001). No entanto, resultados divergentes foram observados no atual estudo, com duração de bloqueio semelhante em ambos os tratamentos. A duração do bloqueio determinado pela lidocaína $(71 \pm 13$ minutos) foi semelhante à relatada em estudos prévios (CRUZ et al., 1997; GASPARINI et al., 2007; CASSU et al., 2010). No entanto, esperava-se que a adição da clonidina resultasse no prolongamento do bloqueio, sobretudo em função da vasoconstricção determinada por esse fármaco no leito vascular peridural (FLACKE et al., 1990), de modo a atenuar o efeito vasodilatador induzido pelo bloqueio simpático decorrente do anestésico local (MENSINK et al., 1987). A inibição da vasodilatação permite a redução na velocidade de captação vascular da lidocaína, de modo a prolongar o bloqueio anestésico (SEGURA et al., 2000). Porém, deve-se ressaltar que a clonidina apresenta alta lipossolubilidade, propriedade molecular que favorece a rápida transferência do fármaco através das meninges para o líquido cefalorraquidiano, determinando curto período de latência e de ação (EISENACH et al., 1989).

Todavia, apesar de o tempo anestésico não ter sido prolongado pela clonidina, a maior dispersão cranial do bloqueio foi confirmada neste estudo, de modo que, em $50 \%$ dos animais, o bloqueio atingiu até a primeira vértebra lombar, enquanto o uso isolado da lidocaína permitiu bloqueio máximo até a quarta vértebra lombar, resultados que concordam com relatos anteriores (GASPARINI et al., 2007; CASSU et al., 2010). A propagação cranial do bloqueio anestésico pode ser obtida mediante a administração de volumes elevados no espaço peridural, bem como em função das propriedades físico químicas dos fármacos empregados (MOORE et al., 1982). No atual estudo, o volume administrado no espaço peridural foi o mesmo em ambos os tratamentos, visto que, no tratamento $\mathrm{C}$, o volume de lidocaína foi reduzido, visando-se a perfazer o volume final de $0,25 \mathrm{ml} \mathrm{kg}^{-1}$, conforme preconizado pela literatura (GASPARINI et al., 2007; CASSU et al., 2010). Dessa forma, sugere-se que a migração cranial do bloqueio possa ser atribuída às características de lipossolubilidade da clonidina (EISENACH et al., 1987), corroborando resultados prévios descritos em ovinos (DE ROSSI et al., 2006).

\section{CONCLUSÃO}

Conclui-se que a adição da clonidina à lidocaína no espaço peridural promove bloqueio anestésico mais cranial em relação ao uso isolado da lidocaína. Paralelamente, a clonidina induz bradicardia em cães saudáveis, além de não prolongar a duração do bloqueio sensitivo.

\section{FONTES DE AQUISIÇÃO}

a - Xilestesin $2 \%$ com vasoconstritor, Cristália, Itapira, Brasil.

b - Clonidin, Cristália, Itapira, Brasil.

c - Acepran 0,2\%, Univet, São Paulo, Brasil.

d - Isorane, Cristália, Itapira, Brasil.

e - Agulha hipodérmica, 30x7, BD, São Paulo, Brasil.

f - Eletrocardiógrafo CARDIOTEST EK 5, São Paulo, Brasil.

g - Doppler 841-A, Parks Medical Electronics, Las Vegas, EUA.

h - Termômetro digital, BD, São Paulo, Brasil.

i - 248-pH Blood Gas Analyser, Ciba Corning Diagnostics, Medfield, MA, USA.

\section{COMITÊ DE ÉTICA}

Este estudo foi aprovado pelo Comitê de Ética em Pesquisa (CEP) da instituição de origem (protocolo n.115/04) e realizado de acordo com os princípios éticos na experimentação animal.

\section{REFERÊNCIAS}

BOPP, S. Clonidina ou sufentanil epidural em cães submetidos à ovário-histerectomia. 2004. 87f. Dissertação (Mestrado em Cirurgia Veterinária) - Curso de Pós-graduação em Medicina Veterinária, Universidade Federal de Santa Maria, RS.

BROCK, N. Acepromazine revisited. Canadian Veterinary Journal, v.35, p.458-459, 1994.

BRONDANI J.T. et al. Analgesia epidural com clonidina ou romifidina em cães submetidos à cirurgia coxofemoral. Arquivo Brasileiro de Medicina Veterinária e Zootecnia, v.56, p.175-182, 2004.

Ciência Rural, v.40, n.10, out, 2010. 
CASSU, R.N. et al. Anestesia epidural com lidocaína isolada e associada ao fentanil para realização de ovário-salpingohisterectomia em cadelas. Arquivo Brasileiro de Medicina Veterinária e Zootecnia, v.60, p.825-831, 2008. Disponível em: <http://www.scielo.br/scielo>. Acesso em: 15 dez. 2009. doi: 10.1590/S0102-09352008000400008.

CASSU, R.N. et al. Lidocaína com vasoconstrictor isolada e associada ao fentanil via peridural em cães. Ciência Rural, v.40, p.580-586, 2010. Disponível em: <http://www.scielo.br/ scielo>. Acesso em: 22 abr. 2010. doi: 10.1590/S010384782010005000016 .

CLEMENTY, J. et al. Study of the electrophysiologic properties of clonidine administered intravenously. Journal of Cardiovascular Pharmacology, v.8, p.24-29, 1986.

CRUZ, M.L. et al. Epidural anaesthesia using lignocaine, bupivacaine or a mixture of lignocaine and bupivacaine in dogs. Journal of Veterinary Anaesthesia, v.24, p.30-33, 1997.

DE KOCK, M. et al. Epidural clonidine used as the sole analgesic agent during and after abdominal surgery. A dose-response study. Anesthesiology, v.86, p.285-292, 1997.

DE NEGRI, P. et al. How to prolong postoperative analgesia after caudal anaesthesia with ropivacaine in children: S-ketamine versus clonidine. Paediatric Anaesthesia, v.11, p.679-683, 2001 .

DE ROSSI, R. et al. Clinical evaluation of clonidine added to lidocaine solution for subarachnoid analgesia in sheep. Journal of Veterinary Pharmacology and Therapeutics, v.29, p.113-119, 2006.

EISENACH, J.C. et al. Epidural clonidine produces antinociception but not hypotension, in sheep. Anesthesiology, v.66, p.496-501, 1987.

EISENACH, J.C. et al. Epidural clonidine analgesia in obstetrics: sheep studies. Anesthesiology, v.70, p.51-56, 1989.

EISENACH, J.C.; GRICE, S.C. Epidural clonidine does not decrease blood pressure or spinal cord blood flow in awake sheep. Anesthesiology, v.68, p.335-340, 1988.

FLACKE, J.W. et al. Hemodynamic effects of dexmedetomidine, an alpha 2-adrenergic agonist, in autonomically denervated dogs. Journal of Cardiovascular Pharmacology, v.16, p.616-623, 1990.

GASPARINI, S.S. et al. Anestesia epidural com ropivacaína, lidocaína ou associação de lidocaína e xilazina em cães. Efeitos cardiorrespiratório e analgésico. Ciência Rural, v.37, p.418424, 2007. Disponível em: 〈http://www.scielo.br/scielo>. Acesso em: 10 dez. 2006. doi: 10.1590/S0103-84782007000200019.
GHIGNONE, M. et al. Hemodynamic effects of clonidine injected epidurally in halotane anaesthetized dog. Canadian Journal of Anaesthesia, v.34, p.46-50, 1987.

IVANI, G. et al. Ropivacaine-clonidine combination for caudal blockade in children. Acta Anaesthesiologica Scandinavica, v.44, p.446-449, 2000.

MATSUKAWA, T. et al. Heat flow and distribution during epidural anesthesia. Anesthesiology, v.83, p.961-967, 1995.

MENSINK, F.J. et al. Dose-response relationship of clonidine in tetracaine spinal anesthesia. Anesthesiology, v.67, p.717$721,1987$.

MOORE, R.A. et al. Dural permeability to narcotics: in vitro determination and application to extradural administration. British Journal of Anaesthesia, v.54, p.1117-1128, 1982.

NOLTE, I. et al. Cardiovascular effects of epidural blocks in dogs. Journal of Small Animal Practice, v.24, p.17-21, 1983.

ROSO, N.C. et al. Hemodynamic and renal effects of high epidural clonidine doses in dogs. Revista Brasileira de Anestesiologia, v.55, p.308-315, 2005. Disponível em: <http://www.scielo.br/scielo>. Acesso em: 09 dez. 2009. doi: 10.1590/S0034-70942005000300007.

SEGURA, I.A. et al. Antinociceptive and motor-blocking action of epidurally administered IQB-9302 and bupivacaine in the dog. Regional Anesthesia and Pain Medicine, v.25, p.522528,2000

SINCLAIR, M.D. A review of the physiological effects of $\alpha 2$ agonists related to the clinical use of medetomidine in small animal practice. Canadian Veterinary Journal, v. 44, p.885$897,2003$.

SISTO, R.K.A. Efeitos da administração peridural de neostigmina associada ou não à clonidina sobre a concentração alveolar mínima do isoflurano em cães. 2010. 115f. Dissertação (Mestrado em Anestesiologia Experimental) - Curso de Pós-graduação em Anestesiologia, Universidade Estadual Paulista, Botucatu, SP.

TIBIRIÇA, E. et al. An imidazoline-specific mechanism for the hypotensive effect of clonidine: a study with yohimbine and idazoxan. Journal of Pharmacology and Experimental Therapeutics, v.256, p.606-613, 1991.

TOUSSAINT, C. et al. Electrophysiological effects of intravenous clonidine on sinus node function and conduction in man. Journal of Cardiovascular Pharmacology, v.6, p.5660,1984

ZAR, J. H. Biostatistical analysis. New Jersey: PrenticeHall Upper Saddle River, 1996. 718p. 